\title{
High Frequency Simulation of Resonant Tunneling Diodes
}

\author{
Wan-Rone Liou and Patrick Roblin, Member, IEEE
}

\begin{abstract}
The small and large-signal response of the resonant tunneling diode at high-frequencies is studied using a quantum simulator. The Poisson and Schrödinger equations are solved self-consistently for each harmonic using the harmonic balance technique. This ensures that the total current, consisting of the displacement plus conduction currents, is conserved across the device for each harmonic. The RTD exhibits an increased capacitance in the negative differential conductance (NDC) region in agreement with experimental data. As recently proposed this capacitance increase results from the formation of an emitter well capacitor when the well discharges. The derivation of the RTD capacitance from a quasi-static analysis using the differential variation of the dc charge in the RTD is shown to be not applicable because the RTD well charges through the cathode but discharges through the anode. The frequency dependence of the conductance and susceptance is similar to reported experimental data. A large frequency dependence of the admittance is only observed when the RTD is biased in the negative differential conductance (NDC) region. These calculations predict an effective reduction of the RTD conductance and capacitance at high-frequency in the NDC region. This effect can be modeled using a quantum inductance in series with the negative resistance of the RTD as recently proposed. Due to the simultaneous reduction of both the conductance and the capacitance at high-frequency in the NDC region the maximum frequency of oscillation does not differ much from its estimate using the low frequency conductance and capacitance. The large-signal response at high-frequency of an RTD biased in the negative differential region is also presented in this paper. The large-signal negative-conductance is shown to decrease with both increasing frequency and ac voltage.
\end{abstract}

\section{INTRODUCTION}

T THE resonant tunneling diode (RTD) which was first demonstrated by Chang et al. is a high-speed device [1]. Negative differential conductance (NDC) with an intrinsic response time of order $0.1 \mathrm{ps}$ has been measured experimentally [2]. With this remarkable high speed performance, the RTD has a wide variety of applications for microwave and millimeter wave oscillation circuits [3], [4]. The fabrication of RTD's is made possible thanks to modern growth techniques which permit the fabrication of high quality one-dimensional heterostructures. Both the optimization of the growth techniques and the use of novel materials will permit the development of resonant tunneling diodes with higher peak-to-valley ratios and with higher maximum frequencies of oscillation [5] and output power. For example, Brown and his colleagues [3] recently

Manuscript received May 6, 1993; revised February 5, 1994. The review of this paper was arranged by Associate Editor P. M. Solomon. This work was supported in part by a grant by the CRAY Corporation and in part by the Ohio State University.

The authors are with the Department of Electrical Engineering, The Ohio State University, Columbus, OH 43210-1272 USA.

IEEE Log Number 941847. observed oscillations in InAs/AlSb RTD's at frequencies up to $712 \mathrm{GHz}$ improving their previous report of oscillations in $\mathrm{GaAs} / \mathrm{AlAs}$ RTD at frequencies up to $420 \mathrm{GHz}[6]$.

A comprehensive simulation of the high frequency response of resonant tunneling diodes would be useful for both the optimization of the RTD structure and the design of optimal oscillation circuits. The maximum frequency of oscillation of the resonant tunneling diode is believed to be limited by various time constants such as the RC time delay, the resonanttunneling traversal time and the depletion-layer transit time [6]. In particular the capacitance of the RTD has an important effect on the maximum frequency of oscillation [7] and should be optimized. However, the measurement of the RTD capacitance in the NDC region [8]-[13], [45] is difficult (because RTD's are usually unstable) and existing theories of the capacitance voltage characteristics conflict (see review below). It would therefore be useful to develop a simulator calculating the RTD capacitance from first principles. It is also presently very difficult to measure the frequency response of RTD's at very high frequencies (above $200 \mathrm{GHz}$ ). A realistic ac-signal simulator would permit us to estimate the frequency response for arbitrarily high frequencies.

Note that in this paper we limit our study to RTD's which are dc stable in the NDC region. This is not a limitation since a stable intrinsic RTD can usually be defined even for RTD's which are dc unstable in the NDC region. Indeed Liu [14] and Belhadj et al. [15]) have clearly shown, both theoretically and experimentally, that the plateaus and hysteresis observed in the NDC region of the I-V of dc unstable RTD's resulted from the interaction of a stable intrinsic RTD with its external circuit consisting of the contact resistances $R_{s}$, the parasitic lead inductance $L_{\text {lead }}$ and the load resistance all in series between the voltage source and the RTD. Jensen and Buot [16] have proposed an alternate mechanism $(2.5 \mathrm{THz}$ intrinsic oscillations) which has not yet been experimentally observed. Note that a stable I-V can usually be measured [17] for a given RTD structure if care is taken to use a small enough diode area in order to satisfy the stability criteria given by Hines [18]. In summary our present study is concerned with intrinsic RTD's (no parasitic lead inductance and contact resistance) which are dc stable and hysteresis-free.

The literature on time-dependent resonant tunneling is extensive. The approaches reported so far have partitioned this problem in two parts: the calculation of the RTD capacitance and the calculation of the frequency response of the intrinsic RTD. The intrinsic frequency response of the RTD was first numerically calculated using the Wigner distribution by Frensley 
[19], Kluksdahl [20], and Mains and Haddad [21]. Analytical expressions were then derived by Chen and Ting [22], Kislov and Kamenev [23] and Fu and Dudley [24]. All these studies established that the negative differential resistance $R$ remains constant and the susceptance essentially null for frequencies below the RTD intrinsic frequency $2 \Gamma / \hbar$ where $\Gamma$ is the energy width of the resonant level. This intrinsic RTD frequency is typically several THz. These studies [19], [20], [22], [24] established that the susceptance of the intrinsic RTD biased in the NDC region is negative at low frequencies and therefore resembles an inductance [19]. However the result of reference [21] differed by a sign and therefore predicted a capacitive behavior. Recently Frensley [25] reverted his interpretation to obtain agreement with reference [21]. We believe that both references committed a simple interpretation error [25] and [21] and that their calculations also predict an inductive behavior. Our own calculation is supportive of the inductive behavior. At the request of the reviewers we have added in appendix a discussion on this subject to justify our approach.

Brown et al. have obtained an improved fit of the power of resonant tunneling diodes by using an inductor $L_{Q W}$ in series with the negative differential resistance $R$ [27]. They proposed that this inductance was associated with the resonance width $I$ of the RTD transmission coefficient. They heuristically derived that this inductance was given by $L_{Q W}=\hbar R / \Gamma$. A similar expression with a factor 2 difference is easily derived from the analytical admittance obtained by Kislov and Kamenev [23]. Fu and Dudley analytically verified that this expression was valid under certain bias conditions [24]. Scattering is believed not to modify $\Gamma$ [26], [27] but should increase this inductance since it increases R (i.e., decreases the NDC, see discussion in the conclusion). It results from these analyses that resonant tunneling is a very fast process and that the practical frequency limitation of RTD's essentially arises from the RTD capacitance.

Several approaches for calculating the capacitance of RTD's have been reported in recent years. A simple estimate of the RTD real capacitance can be obtained with a parallel-plate model $\epsilon / L$. Since the negative charges in the accumulation and well regions image the charge in the depletion region, Luryi [29] proposed to use the barrier width for $L$ to define the RTD capacitance. The barrier width was found to greatly overestimate the RTD capacitance and $f_{\text {MAX. This prompted the }}$ development of the sequential tunneling theory [29] which has been demonstrated to be an alternate description of scattering assisted tunneling but not an alternative mechanism for it (see Zohta [30] and reference therein). More complicated models involving multiple capacitances were then proposed by Luryi [31] and adopted by Genoe et al. [12] to derive the capacitance voltage characteristic of the RTD. These models are based on the assumption that the electrons completely thermalize inside the double barrier. We assume in the RTD's simulated here that thermalization in the well is negligible. A discussion on this topic is included in the conclusion.

An improved fit of the capacitance using the parallel-plate capacitance model was obtained by Brown et al. (see $\mathrm{Hu}$ and
Stapleton [33]) by using

$$
L=L_{B, E}+L_{W}+L_{B, C}+L_{\text {depletion }}\left(V_{D}\right)
$$

where $L_{B, E}$ and $L_{B, C}$ are the barrier width on the emitter and collector side, $L_{W}$ the well width and $L_{D e p l e t i o n}$ the depletion width on the collector side. Note that this model is consistent with the fact that the electrons penetrate and accumulate in the emitter spacer (cathode side) but do not penetrate the collector spacer (anode side). The depletion width on the collector side should therefore include the collector spacer width. This model accounts for the gradual decrease of the RTD capacitance due to the increasing depletion width in the collector side with increasing bias voltage.

Another approach for calculating the capacitance of RTD's was proposed by $\mathrm{Hu}$ and Stapleton [32] and [33]. They evaluate the capacitance $C\left(V_{D}\right)$ at a bias voltage $V_{D}$ originating from the charge $Q_{w}\left(V_{D}\right)$ stored in the quantum well and the accumulation charge $Q_{a}\left(V_{D}\right)$ at the emitter

$$
C\left(V_{D}\right)=\frac{d Q_{w}\left(V_{D}\right)}{d V_{D}}+\frac{d Q_{a}\left(V_{D}\right)}{d V_{D}}
$$

The displacement contribution introduced by the accumulation charge $Q_{a}\left(V_{D}\right)$ in the emitter gives the parallel plate capacitance of (1). When the resonance in the well is quenched the decrease of $Q_{w}$ introduces a negative capacitance component which, if scattering is small, could overcome the depletion capacitance $\epsilon / L$. Their work emphasizes the importance of the charge in the well. Unfortunately we shall see that cannot be applied to the RTD because in the RTD the update of the charge $Q_{w}$ can either be achieved by electrons injected at the cathode or electrons collected at the anode, unlike a true capacitor where the electrons cannot cross the dielectric.

The approaches described above [19]-[24] for calculating the frequency response of RTD's have decoupled the calculation of the electron current and displacement current. In reality the transport in the RTD is, among other things, spacecharge limited and the displacement and electron currents are intimately coupled. Indeed we shall demonstrate that it is not a good approximation to assume that the displacement or electron currents are individually constant across the RTD. In fact, it is the total (displacement+electron) current which is constant across the RTD. It is therefore necessary to solve selfconsistently Poisson and Schrödinger equations for both their $\mathrm{dc}$ and ac response. Although all the ac simulators described above account for the intrinsic RTD response, none of these ac simulators account for self-consistent effects. An AC simulator accounting for dc self-consistency was recently reported by [28]. However, ac self-consistency is not enforced.

In this paper we present such a quantum simulator. As in [23] and [28], a Fourier series expansion is used to analyze the time-dependent resonant tunneling. Perturbation is not used, however, in order to obtain both the small and large signal response. A harmonic balance approach is implemented to obain the desired self-consistency. First the test resonant-tunneling structures studied are presented in Section II. In Section III, we present the large-signal method used to solve the Schrödinger equation and explain the self-consistent approach used for both the dc and ac analysis. Methods for calculating the capacitance 


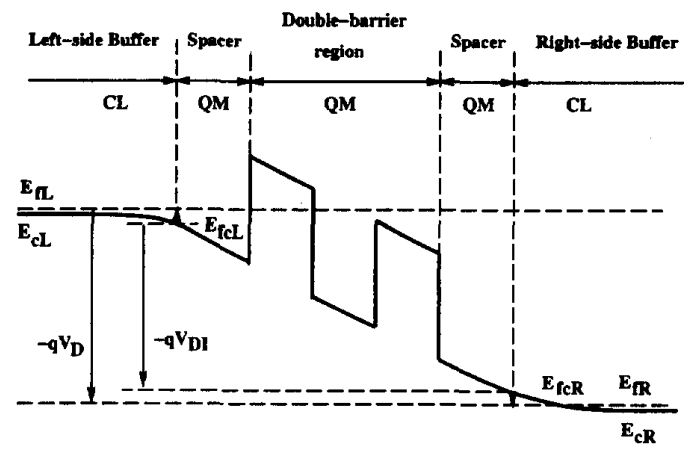

Fig. 1. Band diagram of the test resonant-tunneling structure.

are presented in Section IV. The frequency response of the test RTD and its bias dependence are then presented in detail in Section V. The paper concludes in Section VI with a discussion of the implications and limitations of our simulations.

\section{TEST Resonant TUNNELING StRUCtURE}

To test the time-dependent tunneling simulator proposed below we will first employ a resonant tunneling diode (see Fig. 1) consisting of a conventional undoped AlGaAs/GaAs/AlGaAs double barrier (DB) structure (structure I) with two lightly doped spacers on both sides sandwiched between two strongly doped $G a A s n^{+}$buffers. In structure I, the barrier width and the well width are 6 lattice parameters wide $(\simeq 34 \AA$ ). A barrier height of $0.25 \mathrm{eV}$ corresponding to an $\mathrm{Al}$ mole fraction of 0.3 is used. The band structure of $A l_{m} G a_{1-m} A s$ is modeled using a tight-binding band with an effective mass of $.076+0.1 m$ the electron rest mass. The area of this test device is $4.5 \times 10^{-8} \mathrm{~cm}^{2}$ (the area of one of the diodes which were used for the experimental verification of this work [17]).

As shown in Fig. 1 the voltage $V_{D}$ applied across the resonant tunneling diode Fermi levels $E_{f L}$ and $E_{f R}$ is given by

$$
V_{D}=V_{D I}+E_{f c L}-E_{f c R}
$$

where $V_{D I}$ is the intrinsic voltage appearing across the quantum structure (double barrier and spacer structure) and with $E_{f c L}$ and $E_{f c R}$ the built-in potentials in the buffers supported by the left and right boundary electric fields $F_{l}$ and $F_{r}$. A donor concentration $N_{D}=10^{18} \mathrm{~cm}^{-3}$ is used in the buffers. For this high concentration the donor ionization energy vanishes, and the donor can be assumed to be completely ionized [34]. The I-V characteristic of structure I is shown in Fig. 2.

Another test structure (structure II) considered in this paper is an InGaAs/InAlAs/InGaAs double barriers structure latticed matched on InP. The barrier width is $40 \AA$, the well width $60 \AA$, and the spacer width $135 \AA$. The InGaAs/InAlAs conduction band discontinuity is taken to be $0.5 \mathrm{eV}$. A donor concentration $N_{D}=10^{19} \mathrm{~cm}^{-3}$ is used in the buffers. The area of this test device is also $4.5 \times 10^{-8} \mathrm{~cm}^{2}$. The I-V characteristic of structure II is shown in Fig. 3.

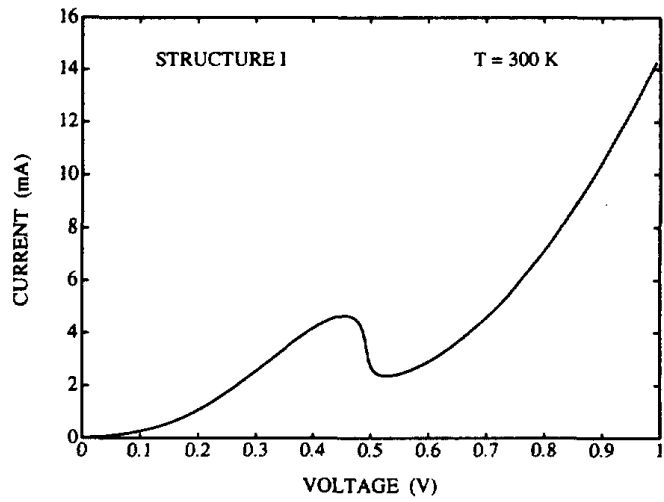

Fig. 2. Current versus applied voltage $V_{D}$ for structure I.

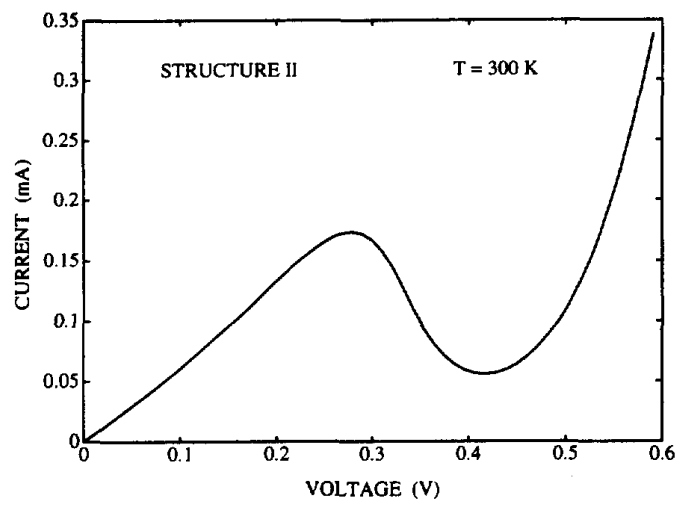

Fig. 3 Current versus applied voltage $V_{D}$ for structure II.

\section{THE AC Simulator}

\section{A. Time-Dependent Tunneling Theory}

Time-dependent tunneling in an RTD is obtained here by solving the one-electron Schrödinger equation

$$
i \hbar \frac{\partial \Psi(x, t)}{\partial t}=\left[H_{o}(x)-e V(x, t)\right] \Psi(x, t)
$$

where $H_{o}(x)$ is the crystal Hamiltonian and $V(x, t)$ the electrostatic potential.

In this study of time-dependent tunneling, we only consider harmonic ac potentials of the form

$$
V(x, t)=V_{o}(x)+\sum_{p} V_{p}(x) \cdot e^{j p \omega t}
$$

with $V_{p}(x)=V_{-p}^{*}(x)$.

Our approach for the electron problem is based on the Generalized Wannier Picture [35] which expands the electron wave function $|\Psi\rangle$ in terms of the Generalized Wannier functions

$$
\left|\Psi>=\sum_{n=-\infty}^{\infty} f(n, t)\right| \mathbf{n}>
$$

where $f(m, t)$ is the Wannier envelope function and $m$ the lattice site index. We limit our analysis here to the tight- 
binding bandstructure

$$
E(\mathbf{k}, n)=E_{c}(n)+\frac{\hbar^{2}\left(k_{y}^{2}+k_{z}^{2}\right)}{2 m^{*}}+\frac{\hbar^{2}}{m^{*} a^{2}}\left(1-\cos k_{x} a\right)
$$

where $k_{x}, k_{y}$, and $k_{z}$ are the electron wave vectors along the $x, y$, and $z$ directions ( $x$ is perpendicular to the heterostructure), $m^{*}$ the effective mass of the semiconductor, $a$ the lattice parameter and $E_{c}(n)$ the conduction band reference energy at the position $x=n a$.

The electron wavefunction $f(m, t)$ along the $x$ axis is then a solution of the Wannier difference equation [36]

$$
\begin{aligned}
& i \hbar \frac{\partial}{\partial t} f(n, t)=-\frac{A}{2} f(n-1, t)-\frac{A}{2} f(n+1, t) \\
& \quad+\left(E_{c}(n)+A-e V_{o}(n a)-e\left[\sum_{p} V_{p}(n a) \cdot \exp (j p \omega t)\right]\right) \\
& \quad f(n, t)
\end{aligned}
$$

where $A$ is $\hbar^{2} /\left(m^{*} a^{2}\right)$. For simplicity we only present here the case of a uniform band structure. In the simulator, spatially varying band structures are implemented (see [36]) using the effective mass matching techniques developed by Kroemer and Zhu [37].

The envelope equation is solved for its steady state response by expanding the wave function (envelope) in terms of a Fourier series

$$
f(n, t)=\sum_{p} f_{p}(n) \cdot e^{-j \frac{E_{0 x}}{\hbar} t-j p \omega t}
$$

where $f_{p}(n)$ is the harmonic component $p$ of energy $E_{o x}+p \hbar \omega$ of the wavefunction. Substituting (8) in (7) we obtain

$$
\begin{aligned}
& \left(E_{o x}+p \hbar \omega\right) f_{p}(n)= \\
& -\frac{A}{2}\left[f_{p}(n+1)+f_{p}(n-1)\right] \\
& +\left(E_{c}(n)+A-e V_{o}(n a)\right) f_{p}(n) \\
& -e \sum_{m}\left[V_{-m}(n a) \cdot f_{p-m}(n)+V_{m}(n a) \cdot f_{p+m}(n)\right] .
\end{aligned}
$$

The set of (9) for each harmonic $p$ forms a linear system of coupled difference equations which is readily solved numerically. For each electron energy an incident wave with unit amplitude is assumed on the left side for the forward current and right side for the backward current. A flat band is assumed on both sides of the RTD. The reference potential (ground) is set on the emitter side so that the conduction band edge on the collector side varies with the applied ac voltage.
The time-dependent electron current between site $n$ and $n+1$ is then given by the usual summation over incident energy $E_{o x}$ [1] (see bottom of page) where $E_{F, l / r}(t)$ is the left and right Fermi level associated with the forward and backward current $I_{F / B}$ respectively and $T(n, t)$ is a time-dependent transmission coefficient defined as

$$
T(n, t)=\frac{\operatorname{Im}\left[f^{*}(n, t) \cdot f(n+1, t)\right]}{\sin \left(\mathrm{k}_{\mathrm{xo}} \mathrm{a}\right)} .
$$

Note that the time-dependence of the Fermi level originates from the dependence of the Fermi level on the left and right electric fields $F_{l}$ and $F_{r}$ which are time dependent. Timeindependent Fermi levels are obtained as boundary condition if a thick enough buffer region is included in the self-consistent analysis so that the ac fields vanish.

Substitution of the Fourier expansion of the wave function given by (8) in (10) permits us to expand the current in a Fourier series

$$
I_{F / B}(n, t)=I_{o, F / B}(n)+\sum_{p} I_{p, F / B}(n) e^{j p \omega t}
$$

where $I_{o, F / B}(n)$ represents the $d c$ forward and backward current, and $I_{p, F / B}^{*}(n)=I_{-p, F / B}(n)$ represents the $p$ th harmonic current component.

Similarly the electron distribution $\rho(n)$ at each lattice site $n$ is calculated using the following expression (see bottom of page) with $E_{x}\left(k_{x}\right)=A\left(1-\cos k_{x} a\right)$. Again substitution of the Fourier expansion of the wavefunction given by (8) in (13) permits us to expand the electron distribution in a Fourier series

$$
\rho_{F / B}(n)=\rho_{o, F / B}(n)+\sum_{p} \rho_{p, F / B}(n) e^{j p \omega t}
$$

where $\rho_{o}(n)$ represents the $d c$ charge density, and $\rho_{p}(n)=$ $\rho_{-p}^{*}(n)$ represents the $p$ th harmonic charge density. The current and charge derived above for each harmonic $p$ verify the continuity equation

$$
I_{p, F / B}(N+1)-I_{p, F / B}(1)=p \omega \sum_{n=2}^{N} \rho_{p, F / B}(n) .
$$

The numerical algorithm implemented in our quantum simulator was verified to satisfy this conservation equation up to the numerical precision provided by the computer used (16 digits of accuracy).

Since electrons are injected at both the emitter and collector contacts the total electron current $I_{\text {elec,p }}$ for the harmonic $p$

$$
\frac{D k_{B} T}{2 \pi} \frac{e}{\hbar} \cdot \int_{0}^{2 A} T(n, t) \cdot \ln \left[\exp \left(I_{F / B}(n, t)=\frac{E_{F, l / r}(t)-E_{c, l / r}-E_{x}}{k_{B} T}\right)+1\right] d E_{x}
$$

$$
\rho_{F / B}(n, t)=\frac{D k_{B} T}{2 \pi} e \cdot \int_{o}^{\frac{\pi}{a}}|f(n, t)|^{2} \cdot \ln \left[\exp \left(\frac{E_{F, l / r}(t)-E_{c, l / r}-E_{x}\left(k_{x}\right)}{k_{B} T}\right)+1\right] d k_{x}
$$




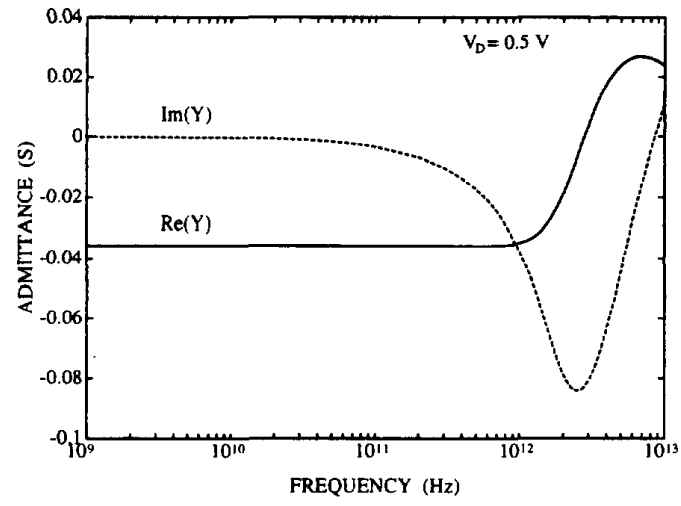

Fig. 4 Small-signal admittance of the RTD (structure I) in the NDC region $\left(V_{D}=0.5 \mathrm{~V}\right)$ plotted versus frequency.

is obtained by substracting the forward (F) and backward (B) currents

$$
I_{\text {elec }, p}=I_{\mathrm{p}, F}-I_{p, B} .
$$

Similarly the total charge is obtained by adding the forward and backward charge

$$
\rho_{p}(n)=\rho_{p, F}+\rho_{p, B} .
$$

As a preliminary test, the quantum ac simulator presented above was verified to reproduce the small-signal frequency response of the intrinsic RTD calculated by [19], [21]-[24] in the absence of self-consistent potential. This is demonstrated in Fig. 4 where the small signal admittance (see next section for a definition) of the RTD (structure I) in the NDC region is plotted versus frequency. As is shown in Fig. 4 the susceptance of the RTD in the NDC region is negative. Since, as it is demonstrated in Appendix, we follow the electrical convention, this implies that the RTD is inductive. This is the so-called quantum inductance [27] which is only observed in the NDC region.

It is verified in Fig. 4 that the device admittance of the intrinsic RTD remains constant up to terahertz frequency. However, we shall see that this does not represent the true frequency response of the RTD since the ac electrostatic potential was not solved self-consistently and the displacement current is not accounted for.

\section{B. Self-Consistent Solution}

As mentioned above, transport in the RTD is, among other things, space-charge limited. As a result the electrostatic potential in the RTD must be calculated self-consistently from the charge distribution. A self-consistent solution of Poisson and Schrödinger equations is known to be required for a more realistic calculation of the dc characteristic of the RTD [38] particularly if undoped spacers are used on both sides of the RTD. Similarly, a self-consistent solution of Poisson and Schrödinger equations is required in order to obtain a more realistic ac characteristic of RTD's.

Like the dc self-consistency, the ac self-consistency is achieved by calculating the electrostatic potential for each harmonic by solving the Poisson equation using the charge distribution obtained from the ac quantum simulator

$$
\begin{gathered}
\frac{d}{d x}\left(\epsilon(x) \frac{d V_{o}(x)}{d x}\right)=\rho_{o}(x)-e N_{D}^{+}(x) \\
\frac{d}{d x}\left(\epsilon(x) \frac{d V_{p}(x, t)}{d x}\right)=\rho_{p}(x, t) .
\end{gathered}
$$

In the simulator, the potential is solved at each lattice site $x=n a$. The boundary conditions applied across the RTD consist of a dc voltage $V_{D}$ and an ac voltage $V_{a c}$ for the first harmonic

$$
V_{D}(t)=V_{D}+V_{a c} \cos (\omega t) .
$$

Higher harmonic voltages are assumed to be shorted outside the device. These boundary conditions are used to calculate all the harmonic components of the RTD current for both the small and large signal response.

For a small ac signal $V_{a c}$, only the first harmonic of the current and internal potential is required. For a large ac signal $V_{a c}$, higher harmonics of both the internal electrostatic potential and the current must be included due to the nonlinearity of the RTD. Theoretically the number of harmonics required can be estimated to be on the order of $P=e V_{a c} /(\hbar \omega)$. Indeed for a flat band collector the amplitude of the harmonics are proportional to the Bessel function $J_{p}(P)$ (see Kislov [23]) which vanishes as $1 / p$ ! for $n>P$.

The iterations proceed as follows. A constant field across the double-barrier and spacer region is used as an initial guess. The Schrödinger equation is solved to calculate the $\mathrm{dc}$ and ac charges densities. Next the Poisson equation is solved and the new dc and ac potentials are then used to solve the Schrödinger equation again. The new and old potentials and charge distributions are averaged to facilitate convergence. This procedure is continued until full consistency between potentials and charge densities is achieved as measured by the convergence of the dc and ac potentials, currents, and charges. An error of $0.1 \%$ requires about 10 iterations outside the NDC region and 20-30 iterations in the NDC region for structure I.

The ac electric field $F_{p}(n a)$ for the harmonic $p$ is then used to calculate the displacement currents $I_{d i s p l, p}$

$$
I_{d i s p l, p}(n)=\epsilon(n) j p \omega F_{p}(n a)=-\epsilon(n) j p \omega \frac{d V_{p}(n a)}{d x} .
$$

The total diode current for each harmonic $p$ is then

$$
I_{\text {total }, p}(n)=I_{\text {elect }, p}(n)+I_{\text {displ }, p}(n) .
$$

The small and large-signal RTD admittance $Y\left(\omega, V_{a c}\right)$ for the first harmonic is then calculated using Kurokawa's definition [39] (see Appendix)

$$
\begin{aligned}
Y\left(\omega, V_{D}, V_{a c}\right) & =\frac{I_{\text {total }, 1}\left(\omega, V_{D}, V_{a c}\right)}{V_{a c}} \\
& =G\left(\omega, V_{D}, V_{a c}\right)+j B\left(\omega, V_{D}, V_{a c}\right)
\end{aligned}
$$

where $G\left(\omega, V_{D}, V_{a c}\right)$ is the device conductance and $B\left(\omega, V_{D}, V_{a c}\right)$ the device susceptance. For small signal excitation $\left(e V_{a c} \ll \hbar \omega\right)$, the quantities $Y, G$ and $B$ are 


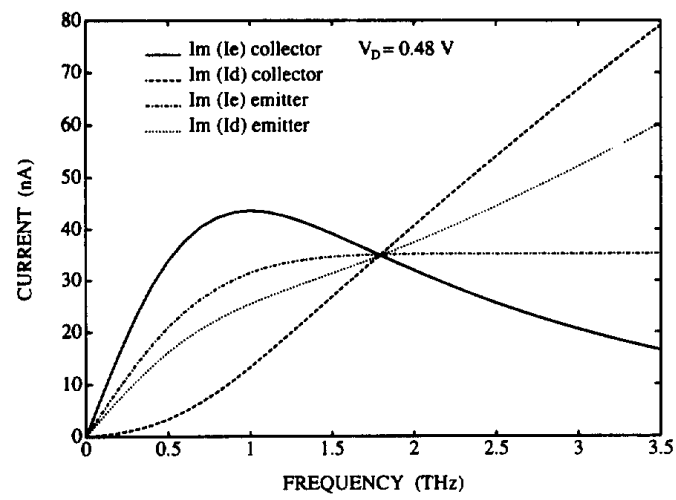

Fig. 5. Imaginary part of the small-signal ac electron and displacement currents versus frequency. The solid and dashed lines represent the electron and displacement currents at the collector buffer (right) while the dashed-dotted and dotted lines represent the electron and displacement currents at the emitter buffer (left).

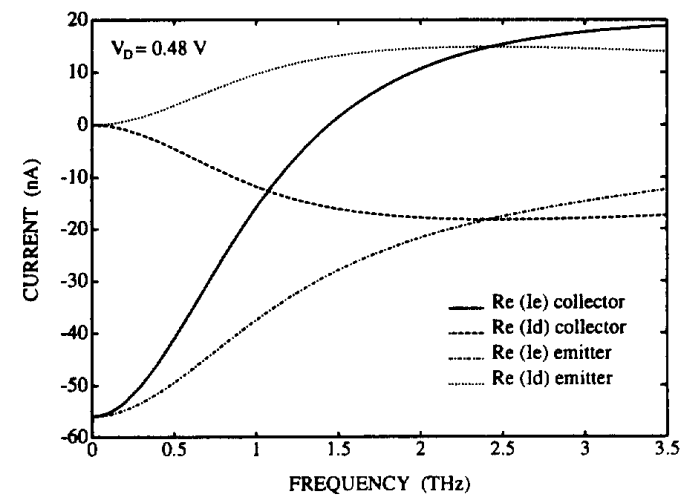

Fig 6. Real part of the ac electron and displacement currents versus frequency. The solid and dash lines represent the electron and displacement currents at the collector buffer (right) while the dashed-dotted and dotted lines represent the electron and displacement currents at the emitter buffer (left).

effectively independent of $V_{a c}$ and only vary with the dc operating point $V_{D}$ and the frequency $\omega$.

Let us now demonstrate the impact of the $\mathrm{dc}$ and ac self-consistent analysis upon the ac current and charge distributions. Consider the RTD of structure I biased in the NDR region $\left(V_{D}=0.48 \mathrm{~V}\right)$ with a small signal ac excitation. Fig. 5 shows a plot versus frequency of the imaginary part of the displacement current $\operatorname{Im}\left[I_{\mathrm{displ}, 1}(n)\right]$ and electron current $\operatorname{Im}\left[I_{\text {elec }, 1}(n)\right]$ at the emitter side (left) and collector side (right) of the RTD for the first harmonic. We define here the emitter and collector sides as the position where the buffer ends and the spacer starts.

Similarly Fig. 6 shows a plot versus frequency of the real part of the displacement current $\operatorname{Re}\left[I_{\text {displ,1 }}(n)\right]$ and electron current $\operatorname{Re}\left[I_{\text {elec, } 1}(n)\right]$ at the emitter side $(n=\mathrm{left})$ and collector side ( $n=$ right) of the RTD for the first harmonic.

Finally Fig. 7 shows a plot versus frequency of both the real part $\operatorname{Re}\left[I_{\text {total, } 1}(n)\right]$ and imaginary part $\operatorname{Im}\left[I_{\text {total }, 1}(n)\right]$ of the total current at both the emitter side $(n=\mathrm{left})$ and collector side ( $n=$ right) of the RTD for the first harmonic.

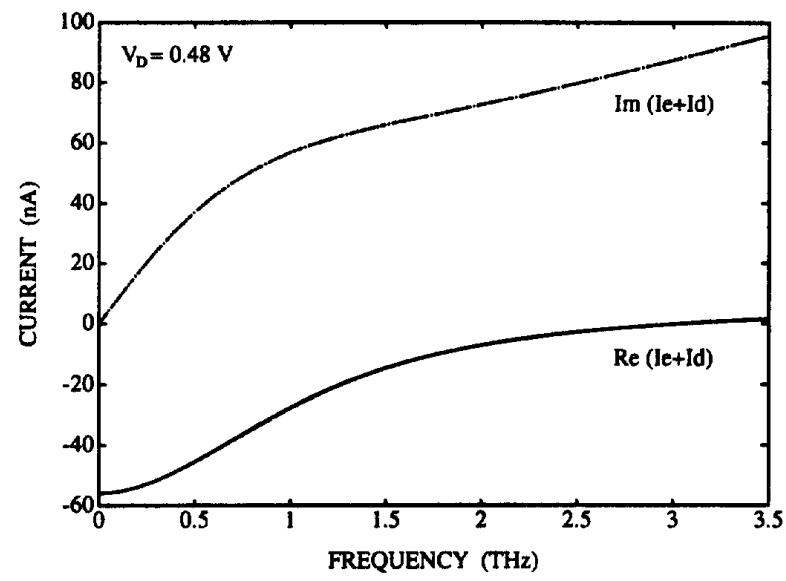

Fig. 7. Real and imaginary part of the small-signal ac current versus frequency. The solid and dashed lines represent the summation of real part of the ac electron and displacement currents at the collector and emitter buffers, respectively, while the dashed-dotted and dotted lines represent the summation of imaginary part of the ac electron and displacement currents at the collector and emitter buffer, respectively.

Clearly the left and right total currents overlap and cannot be distinguished on the plot. This demonstrates the continuity of the total current. On the other hand the displacement and electron currents on the left and right side are clearly different, justifying the need for the self-consistent treatment used for the ac analysis. Indeed the calculation of the displacement and electron currents cannot be decoupled in a rigorous analysis.

\section{Simulation Results}

\section{A. RTD Capacitances}

An examination of Fig. 7 indicates that both the imaginary and real parts of the total current $I_{\text {total, } 1}$ and therefore the conductance $G(\omega)$ and susceptance have a complex frequency dependence.

However, for small frequencies ( $\omega \ll 100 \mathrm{GHz}$ ) the conductance can be approximated by a frequency independent resistor which should be simply the RTD conductance obtained by differentiating the dc IV characteristic of the RTD

$$
G\left(\omega, V_{D}\right) \simeq \frac{d I_{D}}{d V_{D}} \text { for small } \omega .
$$

This is verified in Fig. 8 where we compare the conductance $G\left(V_{D}\right)$ versus dc voltage $V_{D}$ calculated using the dc simulator (plain line) and the ac simulator (* plot) for structure I. A very good agreement is obtained between the dc and ac simulations for the $G-V$ characteristics.

Similarly for small frequency ( $\omega \ll 500 \mathrm{GHz}$ ) we see in Fig. 7 that the RTD susceptance is varying linearly with frequency and is therefore well approximated by a frequency independent capacitance $C$

$$
B(\omega) \simeq \omega C\left(V_{D}\right)
$$

where the capacitance $C$ is the RTD capacitance.

It has been proposed by $\mathrm{Hu}$ and Stapleton [32], [33] that this low-frequency capacitance could be directly calculated from 


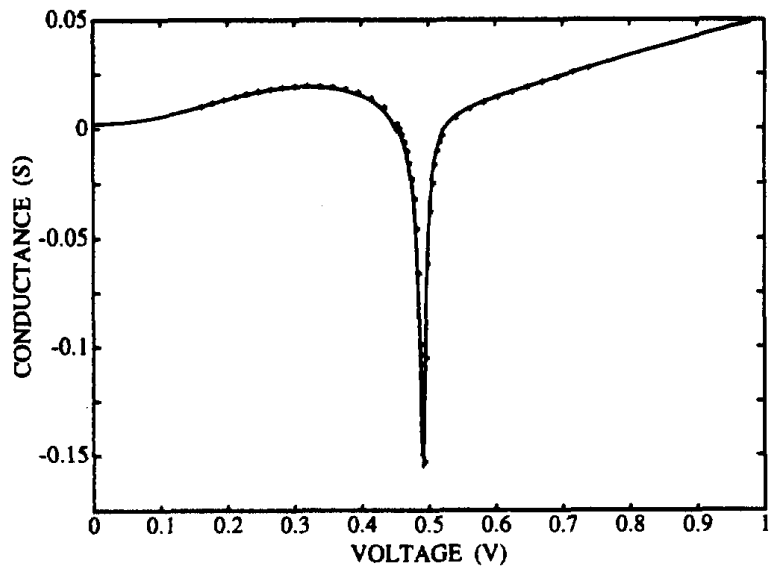

Fig. 8. Comparison of the conductance versus bias voltage calculated from the dc $I-V$ characteristics (plain line) with the small-signal conductance $B$ (star plot) at low frequencies.

the dc characteristic of the RTD with a conventional quasistatic analysis by differentiating the dc charge $Q\left(V_{D}\right)$ of the RTD. A definition of the charge $Q\left(V_{D}\right)$ which accounts for the charge in the well was proposed by $\mathrm{Hu}$ and Stapleton [32]. Let us test here this definition. We know that the dc electron distribution $\rho_{o}(n)$ at each lattice site is given by the sum of the forward and backward charge (see (17))

$$
\rho_{o}(n)=\rho_{o, F}(n)+\rho_{o, B}(n) .
$$

We define $Q_{e, F}$ as the total charge in the RTD supplied by the electron current injected at the emitter

$$
Q_{e, F}(V)=\sum_{n=1}^{N} \rho_{o, F}(n) .
$$

We define $Q_{e, B}$ as the total charge in the RTD supplied by the electron current injected at the collector (right)

$$
Q_{e, B}(V)=\sum_{n=1}^{N} \rho_{o, B}(n) .
$$

We also need to account for the charge stored in the buffer regions. They are calculated from the dc field $F_{o}(n)$ on the left side $(n=1)$ and right side $(n=N)$ using

$$
\begin{gathered}
Q_{b, l}\left(V_{D}\right)=\epsilon(1) F_{o}(1) \\
Q_{b, r}\left(V_{D}\right)=-\epsilon(N) F_{o}(N) .
\end{gathered}
$$

where $Q_{b, l}$ represents the charge in the left side buffer region, and $Q_{b, r}$ represents the charge in the right side buffer region. Summing these charges together permits to verify that space charge neutrality is enforced for the total device

$$
Q_{b, l}(V)+Q_{e, F}(V)+Q_{b, r}(V)+Q_{e, B}(V)=0 .
$$

This is used as a necessary but not sufficient test of convergence in our simulator.

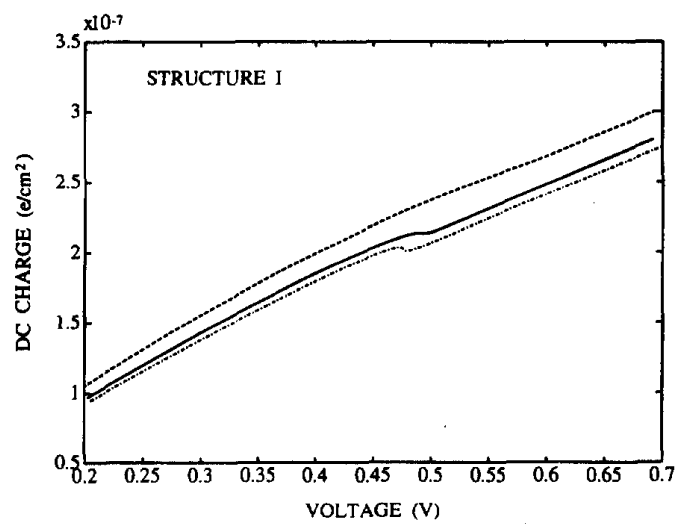

Fig. 9. De charge versus bias voltage for structure $I$ with different spacer width. The solid line represents a spacer width of $34 \AA$, the dash line no spacer, and the dashed-dotted line a spacer width of $45 \AA$.

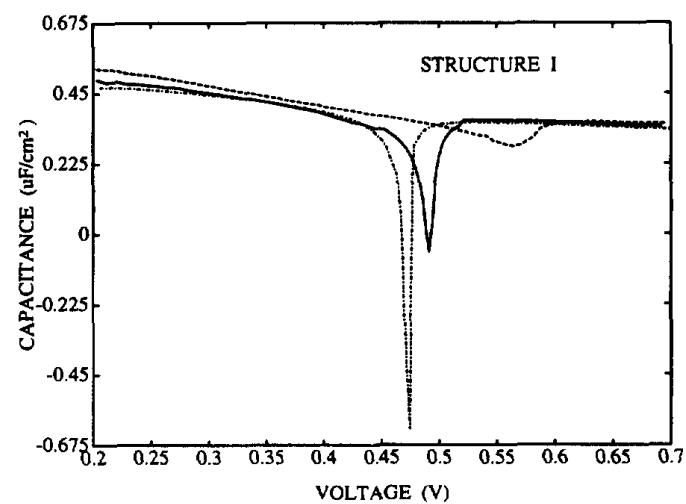

Fig. 10. Quasi-static RTD capacitance versus bias voltage for structure I with different spacer width calculated from the dc (or ac) charge distribution. The solid line represents a spacer width of $34 \AA$, the dash line no spacer, and the dashed-dotted line a spacer width of $45 \AA$.

The charge $Q\left(V_{D}\right)$ is then defined as the total charge supplied by the electron injected at the emitter

$Q\left(V_{D}\right)=Q_{b, l}\left(V_{D}\right)+Q_{e, F}\left(V_{D}\right)=-\left(Q_{b, r}\left(V_{D}\right)+Q_{e, B}\left(V_{D}\right)\right)$

and the RTD capacitance is defined using $\mathrm{Hu}$ and Stapleton definition [32] [33] as

$$
C(V)=\frac{d Q(V)}{d V}=\frac{d\left(Q_{b, l}(V)+Q_{e, F}(V)\right)}{d V} .
$$

In Figs. 9 and 10 the dc $Q-V$ and $C-V$ characteristics are presented for various spacer widths, for structure I. These $Q-V$ characteristics are calculated from the self-consistent dc analysis. It can be seen that the charge increases approximately linearly with the bias voltage, except for a narrow bias range corresponding to the maximum negative differential conductance. In this region the capacitance reaches a minimum. The minimum capacitance is positive in the absence of spacers, nearly null for a $34 \AA$ spacer and negative for a spacer of 45 $\AA$. Similar results were obtained by $\mathrm{Hu}$ and Stapleton [32], [33] for the quantum (well) part of the capacitance. 


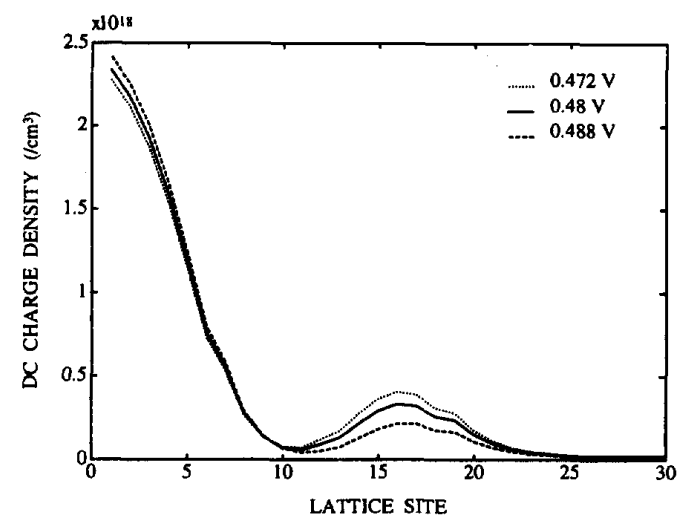

Fig. 11. Distribution of the dc charge across the RTD for three different biasing voltages in the NDC region.

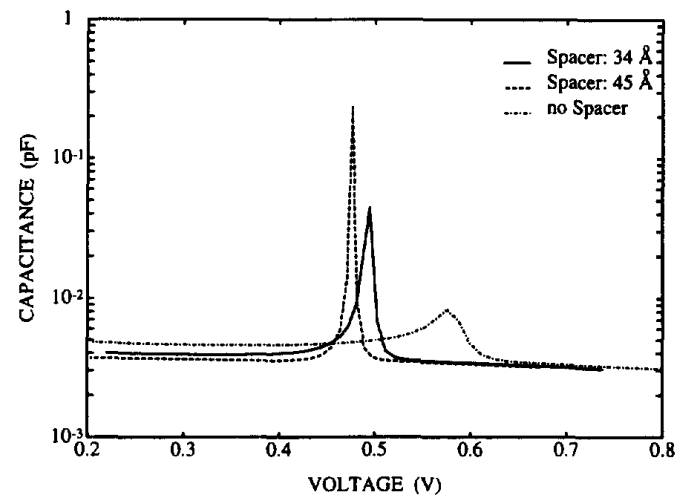

Fig. 12. True RTD capacitance versus bias voltage for structure I with different spacer width calculated from the ac current. The solid line represents a spacer width of $34 \AA$, the dash line no spacer, and the dashed-dotted line a spacer width of $45 \AA$

For a large spacer width the charge decreases in the well for increasing bias can exceed the charge increase in the emitter spacer. This is demonstrated in Fig. 11 where the charge distribution across the RTD is shown for three different voltages around the maximum NDC bias condition. The resulting negative capacitance approximately appears for bias voltages corresponding to the maximum NDC. For structure I an approximately zero capacitance in the NDC region is predicted by the quasi-static analysis for a spacer of length $34 \AA$.

The capacitance versus voltage calculated directly from the ac current using (25) is shown in Fig. 12 for structure I with different spacer lengths. No negative or null capacitance is observed, indeed there is an increase in capacitance. Clearly the capacitance derived from (33) fails to compare with the ac calculation. The reason from the failure of the quasi-static analysis is simply expressed by the continuity (15). The forward charge $Q_{e F}$ can be updated either by the forward current $I_{p, F}(1)$ at the cathode (left) or the forward current $I_{p, F}(N+1)$ at the anode (right). This is demonstrated in Fig. 13 where the imaginary part of the electron and displacement currents are plotted on the emitter (left) and collector (right) sides at a low frequency $(1 \mathrm{GHz})$ as a function of bias voltage.

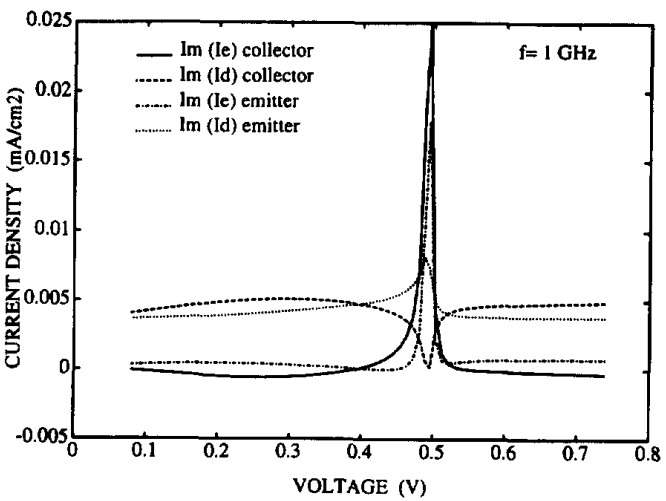

Fig. 13. Imaginary part of the ac electron and displacement currents versus the bias voltage $V_{D}$. The solid and dashed lines represent the electron and displacement currents at the right collector buffer (right) while the dashed-dotted and dotted lines represent the electron and displacement currents at the emitter buffer (left).

The RTD capacitance measured is contributed by the imaginary part of both the electron and displacement currents. The contribution of the displacement current to the capacitor is dominant outside the NDC region (below $0.4 \mathrm{~V}$ and above 0.51 $\mathrm{V}$ ). This indicates that the effective RTD capacitor extends approximately from the emitter buffer to the collector buffer. However for bias voltages inside the NDC region $(0.4-0.51$ V) the contribution of the electron current to the capacitance becomes dominant. Notice also that the displacement current is null on the collector side where all the current is carried by the electron component. This indicates that the charge distribution in the collector side is frozen and that the width of the RTD capacitor has shrunk. This reduction of the RTD capacitor width explains the calculated increase of the RTD capacitance. The RTD capacitor is now being supported by the accumulation layer in the emitter spacer on one side and the accumulation charge in the RTD well on the other side. Indeed we saw in Fig. 11 that the dc charge in the well was varying in opposite direction to the variation of the dc charge in the emitter barrier when the dc applied voltage was varied around the bias voltage of maximum NDC. The existence of this emitter-well capacitance has been recently proposed by Jo et al. [44] to explain the increase of the RTD capacitance and is consistent with Luriy [31] and Genoe et al. [12] models.

From the inspection of the imaginary part of the electron current we can deduct that the forward charge in the well is charged by the electrons injected at the cathode for voltages below $0.4 \mathrm{~V}$ and rapidly collected at the anode when the RTD is biased in the NDC region. Indeed the imaginary part of the electron current is larger on the emitter side than on the collector side for bias voltages smaller than $0.4 \mathrm{~V}$ which permits to charge both the RTD well and the accumulation layer in the emitter spacer. However for bias voltages in the NDC region $(0.4-0.51 \mathrm{~V})$, the situation reverses and the electron current is larger in the collector side than on the emitter side which permits the discharge of the well while the accumulation layer at the emitter keeps charging.

Such a situation is not possible in the gate of an FET where two separate electron currents update the charge on the 


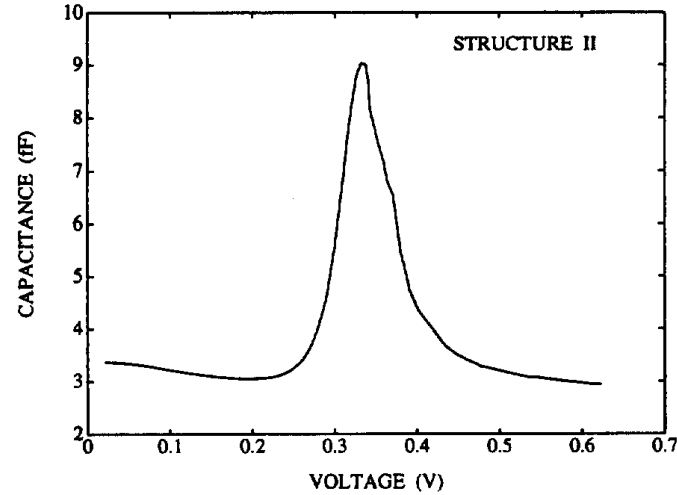

Fig. 14. RTD capacitance versus bias voltage for structure II calculated from the ac current.

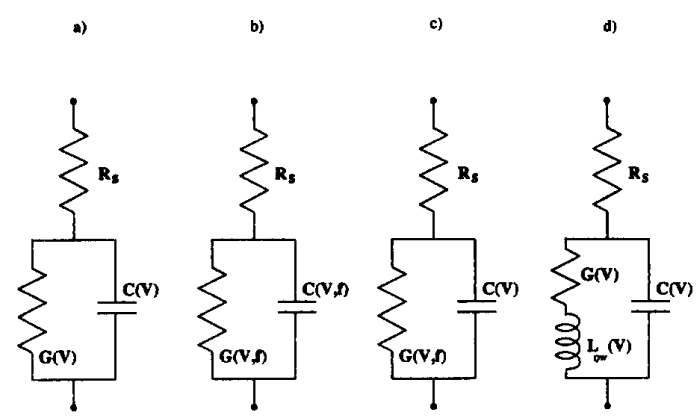

Fig. 15. Equivalent circuits for the RTD structure.

gate and the charge on the channel. A quasi-static analysis is therefore successful with FET's. However, a full selfconsistent ac analysis is required for quantum devices such as the RTD where a single electron current crossing the RTD can create a quantum capacitance by updating in opposite direction the charge at two different locations in the device.

The capacitance versus voltage for structure II is shown in Fig. 14 and is seen to exhibit characteristics similar to those of structure $I$.

The rapid increase of the capacitance in the region of maximum NDC predicted by our ac simulator for both structure I and II is in agreement with the experimental $C-V$ characteristic reported by Genoe et al. [12] for an RTD without a NDC region and by Sammut and Cronin [13] and Boric et al. [45] for a RTD with a NDC region. Indeed in Fig. 1 of [13] the peak of the RTD capacitance is seen to approximately occur for the bias voltages (positive and negative) where the NDC is maximum as predicted by our simulations. Observation of the increase of the RTD capacitance has also been confirmed by Potter et al. for an RTD similar to structure II [17] and more recently by Jo et al. [44].

\section{B. Frequency Response of the RTD}

We have seen that at low frequencies the susceptance of the RTD could be represented by a capacitor and the conductance by a resistor. At higher frequencies the finite velocity of the electrons limits the rate of change of the charge distribution for

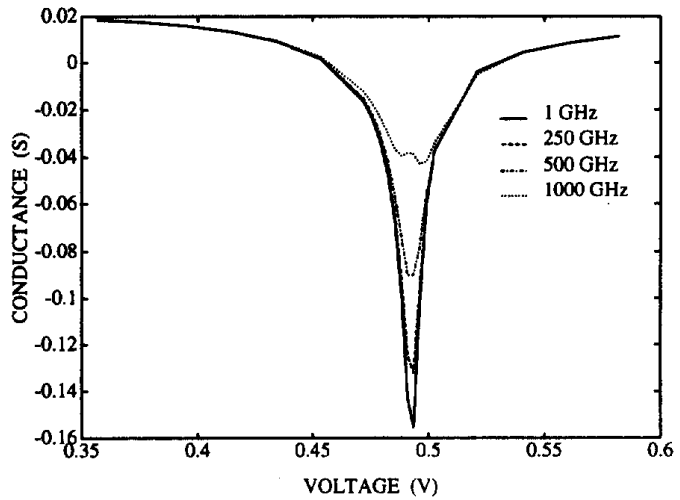

Fig. 16. RTD conductance versus bias voltage for $1 \mathrm{GHz}$ (solid line), 250 $\mathrm{GHz}$ (dashed line), 500 (dashed-dotted line) and $1000 \mathrm{GHz}$ (dotted line).

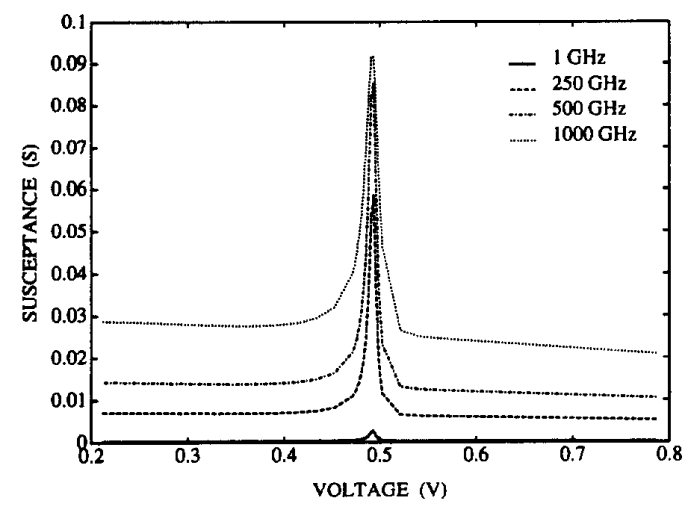

Fig. 17. RTD susceptance versus bias voltage for $1 \mathrm{GHz}$ (solid line), 250 $\mathrm{GHz}$ (dashed line), 500 (dashed-dotted line), and $1000 \mathrm{GHz}$ (dotted line).

a variation of the external ac voltage and the device admittance acquires a more complex frequency dependence.

At low frequencies the RTD is well represented by the circuit shown in Fig. 15(a) where $G\left(V_{D}\right)$ is the RTD conductance, $C\left(V_{D}\right)$ the RTD capacitance and $R_{s}$ the series resistance of the ohmic contact ( $R_{s}=0 \Omega$ in our simulation). A more complex equivalent circuit is needed to represent the complex frequency dependence of the RTD admittance $Y\left(f, V_{D}\right)=$ $G\left(f, V_{D}\right)+j B\left(f, V_{D}\right)$ at higher frequencies. Here we elect to symbolically represent this frequency dependence with a frequency dependent capacitor $C\left(f, V_{D}\right)=B\left(f, V_{D}\right) /(2 \pi f)$ and resistor $R\left(f, V_{D}\right)=1 / G\left(f, V_{D}\right)$ as shown in Fig. 15(b).

Consider Structure I with a spacer length of $34 \AA$. Figs. 16-18 show the real part (conductance) and imaginary part (susceptance) and capacitance of the RTD admittance versus bias voltage for different frequencies. From these figures, we can see that the admittance is strongly frequency dependent in the region of maximum NDC, and the admittance is almost frequency independent outside the NDC region. Similar observations were recently obtained by Mattia et al. [42] from the measured admittance of RTD's.

Note the decrease of the RTD capacitance in Fig. 18 with increasing frequency. Similar experimental data were reported by [12] but for a much lower frequency scale which might be 


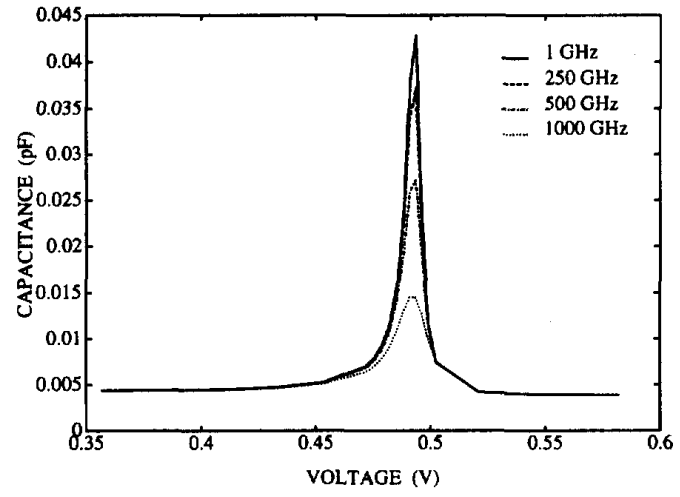

Fig. 18. RTD capacitance versus bias voltage for $1 \mathrm{GHz}$ (solid line), 250 $\mathrm{GHz}$ (dashed line), 500 (dashed-dotted line), and $1000 \mathrm{GHz}$ (dotted line).

due to the fact that their RTD did not exhibit a NDC region.

The reduction of the capacitance and the conductance with high-frequency can be modeled by the equivalent-circuit of Fig. 15(d) proposed by Brown et al. [27] which uses a quantum inductance $L_{Q W}$ in series with the negative conductance of the RTD. Such an equivalent circuit with frequency-independent elements is more physical and is applicable to large-signal simulations.

\section{Maximum frequency of ascillations}

Consider structure I with a spacer length of $34 \AA$. In Figs. 19 and 20 we show the frequency dependence of its conductance and susceptance when this RTD is biased in the NDC region at 0.46 and $0.48 \mathrm{~V}$ (solid line) and in the positive differential region (PDC) at $0.3 \mathrm{~V}$ (dashed line). Clearly the conductance in the NDC region is strongly frequency dependent compared to the PDC region. Furthermore departure of the conductance from its dc value arises at the relative low frequency of 150 $\mathrm{GHz}$ and the $3 \mathrm{~dB}$ break frequency is $800 \mathrm{GHz}$. We obtained a $3 \mathrm{~dB}$ break frequency of approximately $1 \mathrm{THz}$ at $0.48 \mathrm{~V}$ and $2 \mathrm{THz}$ at $0.5 \mathrm{~V}$ in Figs. 7 and 4 , respectively, when we calculated the conductance without self-consistency. Clearly a self-consistent solution of the Schrödinger and Poisson equations is important, as it is seen to slightly reduce the 3 $\mathrm{dB}$ break frequency of the RTD conductance. Since the $3 \mathrm{~dB}$ break frequency is given by $R /\left(2 \pi L_{Q W}\right)$ it results that the quantum inductance $L_{Q W}$ is slightly increased.

Inspection of the susceptance in Fig. 20 reveals that the capacitance in the NDC region decreases at frequencies above $800 \mathrm{THz}$. Let us evaluate the impact of the frequency dependence of both the conductance and susceptance upon the maximum frequency of oscillation.

The maximum frequency is given by the conventional formula [43] (see bottom of page). However, since at high frequencies the conductance and capacitance are frequency

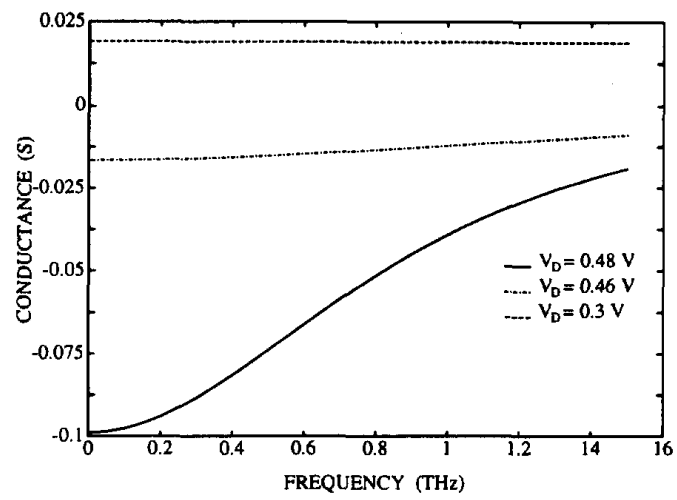

Fig. 19. Conductance versus frequency curve, in the PDC region $V_{D}=0.3 \mathrm{~V}$ (dashed line) and in NDC region 0.46 (dashed-dotted line) and $0.48 \mathrm{~V}$ (solid line)

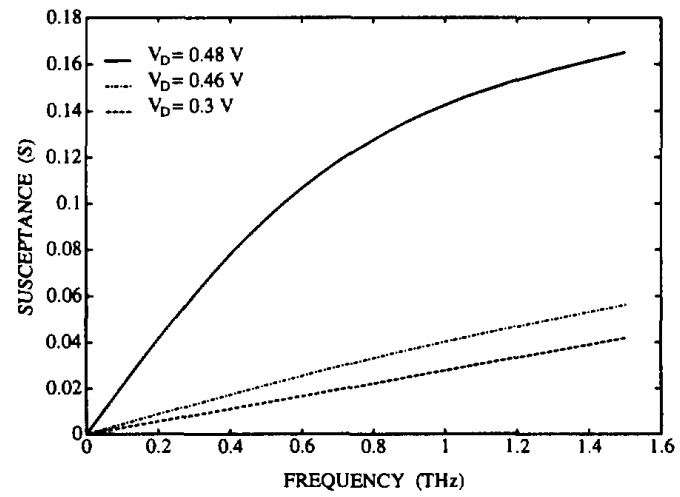

Fig. 20. Susceptance versus frequency curve, in the PDC region $V_{D}=0.3 V$ (dashed line) and in NDC region 0.46 (dashed-dotted line) and $0.48 \mathrm{~V}$ (solid line)

dependent, the expression for $f_{\mathrm{MAX}}$ is now a transcendental equation.

Consider the RTD of structure I with a $34 \AA$ spacer biased in the NDC region at $V_{D}=.48 \mathrm{~V}$. We plot in Fig. 21 the variation of $f_{\operatorname{MAX}}\left(V_{D}=.48 \mathrm{~V}\right)$ with the series resistance $R_{s}$ for the various equivalent circuits shown in Fig. 15. The solid line is calculated with the equivalent circuit 15(a), in which the conductance and capacitance assumed their dc values. The dashed-dotted line is calculated with the equivalent circuit 15 (b), in which both the conductance and capacitance are frequency dependent. The dashed line is calculated with the equivalent circuit $15(\mathrm{c})$, in which the capacitance assumes its $\mathrm{dc}$ value and the conductance is frequency dependent.

From this figure we see that $f_{\mathrm{MAX}}$ is relatively weakly modified by the frequency dependence of the conductance and the susceptance. This surprising result originates from the fact that the capacitance and conductance both decrease with frequency

$$
f_{\mathrm{MAX}}\left(V_{D}\right)=\frac{1}{2 \pi C\left(f_{\mathrm{MAX}}, V_{D}\right)}\left[\frac{-G\left(f_{\mathrm{MAX}}, V_{D}\right)}{R_{s}}-G\left(f_{\mathrm{MAX}}, V_{D}\right)^{2}\right]^{1 / 2}
$$




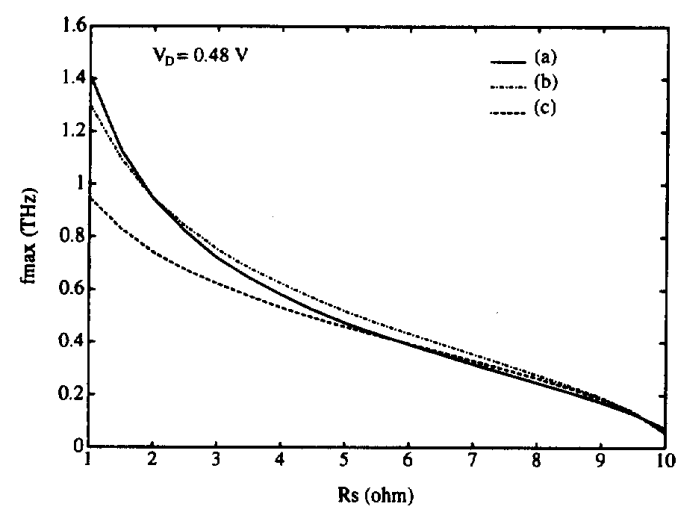

Fig. 21. Maximum oscillation frequency $f_{M A X}\left(V_{D}=0.48 V\right)$ versus the series resistance $R s$

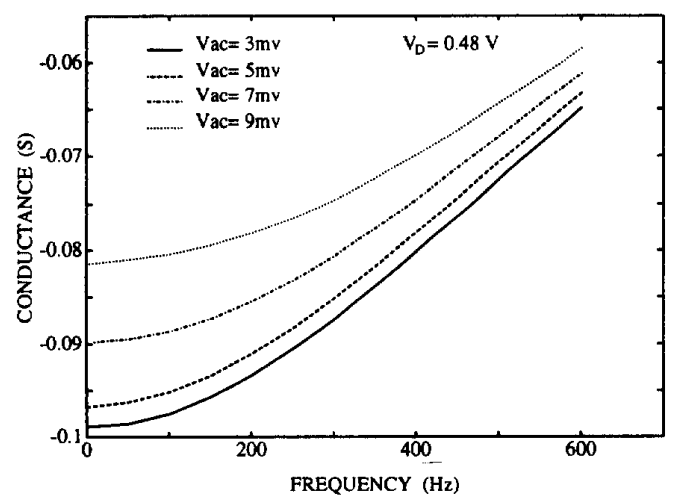

Fig. 22. Conductance $G\left(f, V_{a c}\right)$ at $V_{D}=0.48 \mathrm{~V}$ as a function of frequency for different amplitude of the ac signal $V_{a c}$.

and that a decrease of the conductance decreases $f_{M A X}$ whereas a decrease of the capacitances increases $f_{M A X}$. Indeed when only the frequency dependence of the conductance is accounted for, $f_{M A X}$ will be reduced from $1.4 T H z$ to $0.95 T H z$ when $R_{s}$ is $1 \Omega$ and from $0.98 T H z$ to $0.75 T H z$ when $R_{s}$ is 2 .

Although $f_{M A X}$ is relatively unchanged, the lower $3 \mathrm{~dB}$ break frequency $(800 \mathrm{GHz})$ of the RTD would impact the design of a matching network in an oscillator design at high frequencies.

\section{Large ac Signal Model}

As mentioned earlier our time-dependent tunneling theory can also handle large ac signals. To handle large signal excitations $\left(e V_{a c} \gg \hbar \omega\right)$, more harmonics are required to solve the Schrödinger and Poisson equations self-consistently. Note that the computational time increases with the number of harmonics. Eight harmonics were used for the calculation presented below, and an error of less than $0.1 \%$ was used for the self-consistent calculation.

Consider structure I with a spacer of $34 \AA$. Figs. 22 and 23 show the variation of the large $A C$ signal conductance $G\left(f, V_{a c}, V_{D}=0.48 \mathrm{~V}\right)$ and susceptance $B\left(f, V_{a c}, V_{D}=\right.$ $0.48 \mathrm{~V}$ ) (see (23) for a definition) with the frequency $f$ for

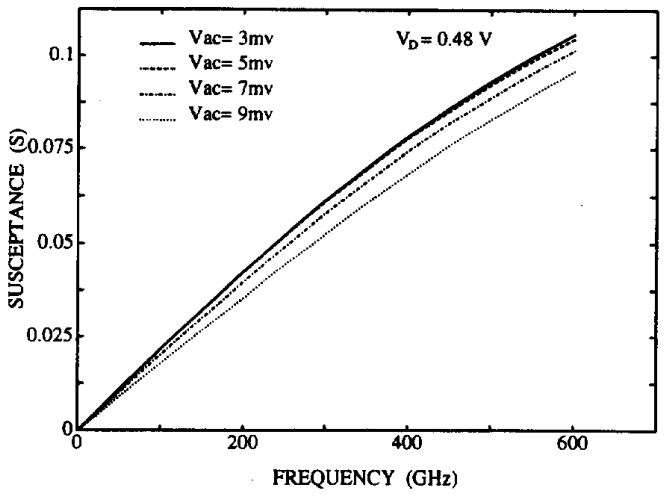

Fig. 23. Susceptance $B\left(f, V_{a c}\right)$ at $V_{D}=0.48 \mathrm{~V}$ as a function of frequency for different amplitude of the ac signal $V_{a c}$.

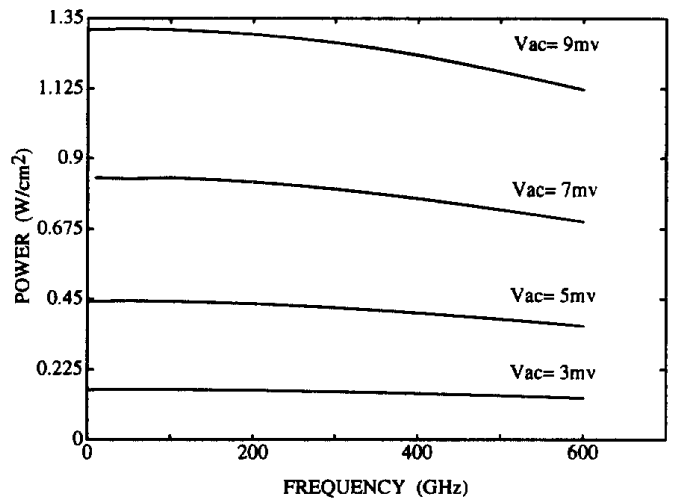

Fig. 24. Ac power generated as a function of frequency for different amplitude of the ac signal $V_{a c}$.

different ac voltage $V_{a c}$. The negative conductance is seen to decrease in amplitude when the amplitude of the ac voltage $V_{a c}$ increases. Similarly to the small-signal response, this negative conductance also decreases in amplitude when the frequency increases. The susceptance is somewhat less dependent on the ac voltage $V_{a c}$ than the conductance. A small reduction of the capacitance is however observed as the RTD sweeps a wider bias region $0.47-0.49 \mathrm{~V}$ around the dc bias point $V_{D}=0.48 \mathrm{~V}$.

Fig. 24 shows the resulting ac power generated $-\operatorname{Re}\left[V_{a c}\right.$. $\left.I_{1}^{*}\right] / 2$ versus frequency for different ac signal levels. The power generated is seen to fall down when the frequency is higher than $300 \mathrm{GHz}$. Note that this is the ac power generated by the intrinsic RTD. The power dissipated by the contact resistance $R_{s}$ is therefore not included. To our knowledge, this is the first estimation of the dependence of the RTD impedance on the large signal ac voltage obtained using a quantum simulation and not an equivalent circuit model.

\section{CONCLUSION}

We have presented a quantum simulator which permits one to study the high-frequency response of resonant tunneling diodes for both small and large-signal excitation. This simulator solves the Poisson and Schrödinger equations selfconsistently using an Harmonic balance technique. This guar- 
antees that the total displacement plus electron current is continuous. The ac self-consistency is shown to reduce several folds the cut-off frequency of the negative differential conductance. However the capacitance is also reduced at high frequencies and the maximum frequency oscillation of the RTD remains essentially the same. The frequency dependence of the conductance and capacitance is however important for the design of the matching network in high-frequency oscillators [6]. The reduction of the negative conductance with large-signal ac excitation is also demonstrated.

The capacitance-voltage $C-V$ characteristic of the RTD is also presented. It is shown that a previous derivation [32], [33] using a quasi-static model is incorrect. The capacitance is seen to increase in the maximum NDC region in agreement with previous experimental data [13] [45]. This is the first $C-V$ reported using a self-consistent ac quantum simulation. A study reporting a peak in the $C-V$ characteristic in the NDC was also reported by Genoe et al. [12]. However, in their work it is assumed that the electrons are fully thermalized in the double barrier and a semi-classical analysis is used. Their model and our model are indeed at the opposite ends of the spectrum. We assume that scattering is negligible whereas they assume that scattering is so large as to completely thermalize the electron gas in the double barrier. Note that their assumption might be realistic for their device which does not exhibit an NDC region. Their derivation relies therefore on the sequential tunneling theory [29] [31]. Note that the sequential tunneling theory has been recently demonstrated to be an alternate description of scattering assisted tunneling but not an alternative mechanism for it (see [30]).

We have recently reported a study of scattering assisted tunneling in resonant tunneling diodes [36]. In this study we demonstrated the impact of multiple-sequential scattering on the resonant tunneling process and introduced a total transmission coefficient $T_{F}$ which is equivalent to the transmission coefficient $T_{T O T}$ introduced by Zohta [30]. The multiplesequential scattering process we analyzed [36] is in fact a realistic simulation of sequential tunneling. Note however that in our study the scattered electrons cross the double barrier by resonant tunneling. Indeed the phase breaking label usually associated with inelastic scattering processes refer only to the randomization of the phase between the incident and scattered electron waves. Consequently the so called "incoherent tunneling" process is effectively the coherent resonant tunneling process of scattered electrons. Experimental support to this concept has been provided by Tsuchiya et al. [26] who verified that the electron life time in the double barrier was set by the energy width of the transmission peak (scattering not included) even in the presence of strong scattering.

In the RTD's studied in [36], phonon-scattering was not strong enough to require multiple-sequential scattering, only interface roughness scattering required a multiple-sequential scattering analysis. However electron-electron scattering processes were not studied and might become important at resonance when the electron concentration in the double barrier increases. Therefore a partial thermalization by electron-electron scattering (but not in equilibrium with the lattice) at resonance in the double barrier has not yet been ruled out. Electronelectron scattering should also impact the accumulation layer on the emitter side.

The contribution of scattering processes on the intrinsic frequency limitation of RTD should also be further studied. Scattering introduces a broadening of the total transmission coefficient (see [36], [30] for a definition). This broadening reduces the NDC and increases the valley current. Scattering processes should therefore be included in a complete highfrequency simulation of RTD's.

\section{ACKNOWLEDGMENT}

The computer time on the CRAY Y-MP was provided by a grant from the Ohio Supercomputer Center. The authors are also indebted to Prof. Furrukh Khan for letting them use his IBM 6000 . The second author would like to thank David Shupe for his kind hospitality at Allied Signal MTC where the manuscript was completed and where the experimental verification of this theoretical work was pursued [17]. He would also like to thank Drs. Robert Potter and Ayub Fathimulla of Allied Signal MTC for many fruitful discussions on related issues. He is also grateful to Dr. Brown at Lincoln Laboratory MIT for an informative discussion on the quantum inductance effect.

\section{APPENDIX}

There is a certain confusion on the sign of the susceptance of the RTD as calculated by quantum simulators, since [21], [25] obtained the same results as [19], [20], [22]-[24] and our own calculation, but with an opposite sign. The confusion seems simply rooted in the use of two different conventions for defining the admittance. The electrical convention followed in this paper uses a phasor $\exp (j \omega t)$ to define the Fourier series and the admittance. The physics (-) convention use the phasor $\exp (-j \omega t)$.

We want first to state here that the solution of Schrödinger in terms of measurable quantities (defined by an Hermitian operator and therefore real) is independent of the use of the electrical or physical sign convention. Indeed the Schrödinger equation uses a real potential $V(x, t)$ in (7), and consequently the potential $V(x, t)$ is unique. This is true even though the complex term $V_{p}$ introduced in (5) is itself dependent of the sign convention $\exp (j \omega t)$ which is used in this paper. In fact in our simulator we actually expand the potential $V(x, t)$ in terms of $\sin$ and $\cos$

$$
V(x, t)=V_{o}(x)+\sum_{p} V_{C p}\left(\cos p \omega t+V_{S p} \sin p \omega t\right)
$$

where $V_{C p}$ and $V_{S p}$ are uniquely defined.

Similarly the time-dependent current $I_{F / B}(n, t)$ defined by (10) and the charge $\rho_{F / B}(n, t)$ defined by (13) are both real functions of time and space and are therefore uniquely defined and independent of the sign convention $\exp (j \omega t)$ used in (12) and (14). Note that the validity of our definition of currents and charges is supported by the fact that they apply both to $\mathrm{dc}$ and ac and satisfy (15).

Although the choice of the phase convention $\exp (j \omega t)$ or $\exp (-j \omega t)$ does not impact the physical quantity $\left(I_{F / B}(n, t)\right.$ 
and $\left.\rho_{F / B}(n, t)\right)$ calculated from the Schrödinger equation, it will impact the definition of the admittance $Y_{p}( \pm)$ since it is convention to use complex numbers to represent the frequency response.

If we use the electrical $(+)$ convention $i(t)=$ Real $[I \exp (j \omega t)]$ we find the admittance for the harmonic $p$ to be

$$
Y_{p}(+)=\frac{I_{C p}-j I_{S p}}{V_{C p}-j V_{S p}}=\frac{I_{p}}{V_{p}}
$$

where $V_{p}$ is defined by (7). Note that for the electrical (+) convention a negative susceptance is an inductance. This is the approach used in this paper.

If we use now the physical $(-)$ convention $i(t)=$ $\operatorname{Real}[I \exp (-j \omega t)]$ we find

$$
Y_{p}(-)=\frac{I_{C p}+j I_{S p}}{V_{C p}+j V_{S p}}
$$

Because $I_{C p}, I_{S p}, V_{C p}$ and $V_{S p}$ are unique quantities (independent of the sign convention) we obtain

$$
Y_{p}(-)=Y_{p}^{*}(+)
$$

This demonstrates that if we use the physics (-) convention [25], a positive susceptance $\left(\operatorname{Im}\left[Y_{p}(-)\right]<0\right)$ is in fact an inductive response and not a capacitive one. This was the approach and interpretation used by $\mathrm{Fu}$ et al. [24].

This appendix demonstrates that our definition and interpretation of the admittance is consistent with our choice of the electrical convention. Obviously a sign error can always be introduced in programming. However, the theoretical calculations [22]-[24] all concur in predicting an inductive response.

\section{REFERENCES}

[1] L. L. Chang, L. Esaki, and R. Tsu, "Resonant tunneling in semiconductor double barriers," Appl. Phys. Lett., p. 593, 1974

[2] T. C. L. G. Sollner, W. D. Goodhue, P. E. Tannenwald, C. D. Parker, and D. D. Peck, "Resonant tunneling through quantum wells at frequencies up to 2.5 THz," Appl. Phys. Lett., vol, 43, no. 6, pp. 588-590, 1983.

[3] E. R. Brown, T. C. L. G. Sollner, C. D. Parker, W.D. Goodhue, and Chen, "Oscillations up to $420 \mathrm{Ghz}$ in GaAs/AlAs resonant tunneling diodes," Appl. Phys. Lett., vol. 55, no. 17, pp. 1777-1779, 1989.

[4] P.D. Coleman, S. Goedeke, T. J. Shewchuk, P. C. Chapin, J. M. Gering, and $H$. Morkoc, "Experimental study of the frequency limits of resonant tunneling oscillator," Appl. Phys. Lett., vol. 48, pp. 422-424 1986

[5] C. I. Huang, M. J. Paulus, C. A. Bozada, S. C. Dudley, K. R. Evans, C E. Stulz, R. L. Jones, M. E. Cheney, "AlGaAs/GaAs double barrier diodes with high peak-to-valley current ratio," Appl. Phys. Lett., vol. 51, p. 121, 1987.

[6] E. R. Brown, J. R. Söderström, C. D. Parker, L. J. Mahoney, K. M Molver, and T. C. McGill, " Oscillations up to $712 \mathrm{GHz}$ in InAs/AlSb resonant-tunneling diodes," Appl. Phys. Lett., vol. 58, p. 2291, 1991.

[7] D. D. Coon and H. C. Liu, "Frequency limit of double barrier resonan tunneling oscillators," Appl. Phys. Lett., vol. 49, no. 2, pp. 94-96, 1986.

[8] J. M. Gering, D. A. Crim, D. G. Morgan, P. D. Coleman, W. Koop and $\mathrm{H}$. Morkoc, "A small-signal equivalent-circuit model for $\mathrm{GaAs}$ $A l_{x} G a_{1-x} A s$ resonant tunneling heterostructures at microwave frequencies," J. Appl. Phys., vol. 61, no. 1, pp. 271-276, Jan. 1987.

[9] D. Lippens and P. Mounaix "Small-signal impedance of GaAs$A l_{x} G a_{1-x} A s$ resonant tunneling heterostructures at microwave frequency," Electronics Lett., vol. 24, no. 18, pp. 1180-1181, Sept. 1988

[10] M. L. Leadbeater, E. S. Alves, L. Eaves, M. Henini, O. H. Hughes, F. W. Sheard, and G. A. Toombs, "Magnetic field and capacitance studie of intrinsic bistability in double-barrier structures," Superlattices and Microstructures, vol. 6, no. 1, pp. 59-62, 1989.
[11] A. Zarea, A. Sellai, M. S. Raven, and D. P. Steenson, J. M. Chamberlain, M. Henini, and O. H. Hughes, "Impedance analysis of GaAs/Al(Ga)As resonant tunnel diodes in the NDR Region," Electronics Lett., vol. 26, no. 18 , pp. 1522-1523, Aug. 1990

[12] J. Genoe, C. V. Hopf, V. V. Roy, J. H. Smet, K. Fobelets, R. P. Mertens, and G. Borghs, "Capacitances in double-barriers tunneling structures," IEEE Trans. Electron Devices, vol. 38, pp. 2006-2012, Sept. 1991.

[13] C. V. Sammut and N. J. Cronin, "Comparison of measured and computed conversion loss from a resonant tunneling device multiplier," IEEE Trans. Microwave and Guided Wave Lett., vol. 2, pp. 486-488, Dec. 1992.

[14] H. C. Liu, "Simulation of extrinsic bistability of resonant tunneling structures," Appl. Phys. Lett., vol. 53, no. 6, pp. 485-486, Aug. 1988.

[15] C. Y. Belhadj, K. P. Martin, S. Ben Amor, J. J. L. Rascol, R. J. Higgins, R. C. Potter, H. Hier, and E. Hempfling, "Bias circuit effects on the current-voltage characteristic of double-barrier tunneling structures: Experimental and theoretical results," Appl. Phys. Lett., vol. 57, no. 1, July 1990.

[16] K.L. Jensen and F.A. Buot, "Numerical Simulation of intrinsic bistability and high-frequency current oscillations in resonant tunneling structures,"' Phys. Rev. Lett., vol. 66, no. 8, pp. 1078-1081, 1991.

[17] R. C. Potter, P. Roblin, W. R. Liou, and Ayub Fathimulla, "Analysis and measurement of the capacitive-voltage characteristic of a resonant tunneling diode," submitted to Appl. Phys. Lett., May 1993.

[18] M. E. Hines, "High-frequency negative-resistance circuit principle of Esaki diode applications," Bell. Sys. Techn. J., vol. 39, pp. 477-513, May 1960.

[19] W. R. Frensley, "Quantum transport calculation of the small-signal response of a resonant tunneling diode," Appl. Phys. Lett., vol. 51, no. 6 , pp. $448-450,1987$

[20] N. C. Kluksdahl, A. M. Kriman, D. K. Ferry, and C. Ringhofer, "Transient switching behavior of the resonant-tunneling diode," IEEE Electron Device Lett., vol. 9, pp. 457-459, Sept. 1988.

[21] R. K. Mains and G. I. Haddad, "Wigner function modeling resonant tunneling diodes with high peak-to-valley ratios," J. Appl. Phys., vol. 64 , no. 10 , pp. $5041-5044,1988$.

[22] L.Y. Chen and C.S. Ting "AC conductance of a double-barrier resonant tunneling system under a dc-bias voltage," Phys. Rev. Lett., vol. 64, no. 26, pp. 3159-3162, 1990.

[23] V. Kislov and A. Kamenev, "High-frequency properties of resonant tunneling devices," Appl. Phys. Lett., vol. 59, no. 12, pp. 1500-1502, 1991.

[24] Yaotian Fu and S. C. Dudley, "Quantum inductance within linear response theory," Phys. Rev. Lett., vol. 70, no. 1, pp. 65-68, Jan. 1993.

[25] W. R. Frensley, "Boundary conditions for open quantum systems," Revue of Modern Physics, vol. 62, p. 764, 1990.

[26] M. Tsuchiya, T. Matsusue, and H. Sakaki, "Tunneling escape rate of electrons from quantum well in double-barrier heterostructures," Phys. Rev. Lett., vol. 59, no. 20, p. 2356, Nov. 1987.

[27] E. R. Brown, C. D. Parker, and T. C. L. G. Sollner, "Effect of quasibound-state lifetime on the oscillation power of resonant tunneling diodes," Appl. Phys. Lett., vol. 54, no. 10, pp. 934-936, Mar. 1989.

[28] Y. Fu and M. Willander, "Response of a semiconductor tunneling structure to a time-dependent perturbation," J. Appl. Phys., vol. 72, no. 8, pp. 3593-3597, 1992.

[29] S. Luryi, "Frequency limit of double-barrier resonant-tunneling oscillators," Appl. Phys. Lett., vol. 47, no. 5, pp. 490-492, 1985.

[30] Y. Zohta, "On the definition of sequential tunneling in a double-barrier resonant tunneling structure," Japan.J. Appl. Phys., vol. 32, part II, no. 2A, pp. L177-179, Feb. 1993.

[31] S. Luryi, "Quantum capacitance devices," Appl. Phys. Lett., vol. 52, no. 6, pp. 501-503, 1988

[32] Y. Hu and Shawn Stapleton, "Quantum capacitance of resonant tunneling diodes," Appl. Phys. Lett., vol. 58, no. 2, pp. 167-169, 1991.

[33] Y. Hu and S. P. Stapleton, "Capacitance of a resonant tunneling diode," Japan. J. Appl. Phys., vol. 31, pp. 23-25, 1992.

[34] B. Vinter, private communication.

[35] P. Roblin and M. W. Muller, "Spatially varying bandstructures," Phys. Rev. B, vol. 32, pp. 5222-5230, 1985.

[36] P. Roblin and W.R. Liou, "3D scattering-assisted tunneling in resonant tunneling diodes," Phys. Rev. B, vol. 47, no. 4, pp. 2146-2161, Jan. 1993

[37] H. Kroemer and Qi-Gao Zhu, "On the interface connection rules for effective-mass wave functions at an abrupt heterojunction between two semiconductors with different effective mass," J. Vacuum Sci. Techn., vol. 21, pp. 551-553, 1982.

[38] M. Cahay, M. McLennan, S. Datta, and M. S. Lundstrom, "Importance of space-charge effects in resonant tunneling diode," Appl. Phys. Lett., vol. 50, p. $612,1987$. 
[39] K. Kurokawa, Bell Syst. Tech. J., vol. 48, p. 1937, 1969.

[40] P. Roblin and M. W. Muller, "Time-dependent tunneling and the injection of coherent Zener oscillations,' Semicond. Science and Techn., vol. 1 , no. 3, pp. $218-225,1986$.

[41] P. Roblin, "Bandstructure matching at a heterojunction interface," Superlattices and Microstructures, vol. 4, no. 3, pp. 363-370, 1988.

[42] J. P. Mattia, E. R. Brown, A. R. Calawa, and M. J. Manfra, "Smallsignal admittance and switching measurements of the resonant-tunneling diode,' to be published.

[43] T. C. L. G. Sollner, E. R. Brown, W. D. Goodhue, and H. Q. Le, "Microwave and millimeter-wave resonant tunneling devices," in it Physics of Quantum Electron Devices, Federico Capasso, Ed. New York: Springer, 1990 , chap. 6.

[44] J. Jo, H. S. Li, Y. W. Chen, and K. L. Wang, "Observation of a large capacitive current in a double barrier resonant tunneling diode at resonance," submitted to Appl. Phys. Lett., Nov. 1993.

[45] O. Boric, T. J. Tolmunen, E. Kollberg, and M. A. Frerking, " Anomalous capacitance of quantum well double-barrier diodes," Int.J. Infrared and Millimeter Waves, vol. 13, no. 6, pp. 799-814, 1992.

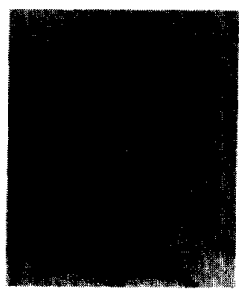

Wan-Rone Liou was born in Penhu, Taiwan, R.O.C., in September 1961. He received the B.S. and M.S. degrees in electrical engineering from National Cheng-Kung University, Taiwan, in 1984 and 1986, respectively, and the Ph.D. degree in electrical engineering from the Ohio State University in 1993

He was a Second Lieutenant of the R.O.C. Navy from 1986 to 1988 . From 1988 to 1989 , he was a chief instructor in the computer center of Wu-Feng Institute of College, Taiwan. He is presently an Associate Professor in the Electrical Engineering Department at Feng Chia University, Taiwan. His current research interests include high-speed device simulation and fabrication, and ASIC design.

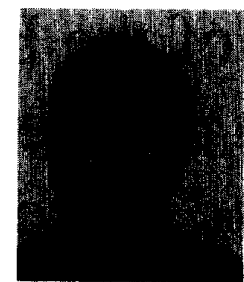

Patrick Roblin (M'85) was born in Paris, France, in September 1958. He received the Maitrise de Physics degree from the Louis Pasteur University, Strasbourg, France, in 1980 and the M.S. and D.Sc. degrees in electrical engineering from Washington University, St. Louis, MO, in 1982 and 1984, respectively. His Ph.D. dissertation studied the synthesis of squeezed Zener oscillations in an NIN semiconductor by time-dependent tunneling.

In 1984 he joined the department of Electrical Engineering at the Ohio State University as an Assistant Professor and became an Associate Professor in 1990. At OSU he has worked on the modeling of the electrical characteristics of MODFET's. In particular he has reported with his students the first exact solution of the velocity-saturated MOSFET/MODFET wave-equation and a new large-signal model topology for FET's which is both charge-conserving and nonquasistatic. He has also developed at OSU an educational microwave laboratory which is used into two senior elective undergraduate courses for the design of passive and active microwave circuits. He has recently reported with one of his student a new type of quantum simulator for the calculation of $3 \mathrm{D}$ scattering-assisted in layered semiconductor heterostructures. He has spent the 1993-94 academic year on professional leave at the Microelectronics and Technology Center at Allied Signal Aerospace in Maryland working on the simulation of resonant tunneling diodes. 